\title{
New Proposed Mobile Telecommunication Customer Call Center Roster Scheduling Under the Graph Coloring Approach
}

\author{
Hasitha Indika Arumawadu \\ School of Computer Science \\ and Technology, Wuhan \\ University of Technology, \\ Wuhan, P. R. China
}

\author{
R. M. Kapila Tharanga \\ Rathnayaka \\ Faculty of Applied Sciences, \\ Sabaragamuwa University of \\ Sri Lanka, Belihuoya, \\ Sri Lanka
}

\author{
D. M. K. N. Seneviratna \\ Faculty of Engineering, \\ University of Ruhuna, Galle, \\ Sri Lanka
}

\begin{abstract}
The call center roster scheduling is one of the significant problem in the mobile telecommunication roster management systems today; especially, creates work plan and allocates working hours for the whole day under the three shifts creates big challenge for the administrators who responsible for creating roster time tables. As a result of assigning employees into roster timetables under the manual scheduling systems create this problem more complicated. This new proposed automated roster scheduling approach developed under the two stages. As an initially, Enhanced Greedy Optimization algorithm is implemented to optimize the hotline roster and compared with other optimization algorithms (Simulated Annealing and Genetic Algorithm). In the Second stage, client server based framework introduced to access and update roster timetables for administrators as well as employees with different access levels.

Keywords: Graph Coloring; Genetic Algorithm; Hotline Roster Scheduling; Mobile Telecommunication; Call Center
\end{abstract}

\section{INTRODUCTION}

A call center is a centralized office used for receiving or transmitting a large volume of requests by telephone. It is a central point from which all customer contacts are managed and provides assistance for the customers to solve their problems related to the service offered by the company. It is generally a part of company's customer relationship management. It can be considered as a big challenge in the modern world today; especially in telecommunication industry under the 24 hour service over the 365 days. Currently, even in most of the reputed companies, spend additional time for creating working schedule manually [1].

The feasible working schedule has mainly impact on the quality of service for their customers as well as their employers. As a result, make unbiased manual scheduling timetable in telecommunication industry has been created more complicated problems today. Moreover, various necessities of customers, different type of qualifications, experiences and specializations, employers and employee requirements, unpredictable incidence and absenteeism and other factors make the problem more complicated.

Before make feasible Customer care hotline roster management system, we should have to concern much more about constraints and requirements going under the different shifts as morning, afternoon and night [2]. Based on customer's feedbacks, the afternoon session has identified as peak and Night and Morning considered as off-peak time. So, many customers take assistance over the phone in Peak time comparing to off-peak time. As a multi ethnic country, especially in Sri Lankan top level companies has been offering different languages in their hotline systems. So, language skills of their customer officers are another requirement we should consider.
Generally, customers can be divided into two categories as loyalty customers and normal customers. To assist loyalty customers, need to assign more experienced and skilled officers to spread balance way to serve better service. Furthermore, some unpredictable incidents and absenteeism should be also consider; especially different religious officers have different holidays, so have to consider religious wise holidays before assigning them into rosters.

In this scenario manual system for scheduling timetable is not effective, flexible and balanced with advanced than in IT technology and efficient optimization algorithms [3]. So, our proposed work, focus on developing a framework based on Graph Coloring algorithm for customer care hotline management system especially for highly populated companies in telecommunication industry in Sri Lanka.

In our approach, client server architecture is proposed for retrieve and manage the employee information. The rest of the paper is organized as follows. Section 2 explains about brief overview of existing solutions with pros and cons. Section 3 explains about proposed work with enhanced greedy optimization algorithm. Section 4 explains about experimental results and Section 5 ends up with conclusion and future work.

\section{LITERATURE REVIEW}

In the last two decades, Graph coloring theories have been widely applied in the literature to find the solutions for real world problems; especially, timetable scheduling, nurse roster scheduling, register allocation, problem of bandwidth allocation and etc [4]. Timetabling is the process of assigning limited resources to a set of events without violating the constraints[5][6].Most of the current proposed solutions either make use of random based optimization algorithms which won't be efficient or applicable only for fully automated scheduling problems. 
Kundu \& Mahato (2007) have done remarkable studies based on Genetic Algorithm (GA) and had deep discussions about the role of data warehouse management system to handle hospital and nurse management information. Based on soft and hard constraints and simulated Annealing, they have described the use of Genetic Algorithm (GA) for solving NSP under the three different levels [7]. Bard \& Purnomo (2005) reported a same kind of methodology to solve the nurse roster problem using column generation. This sub problem was formulated as a shortest path problem with resource constraints, where each possible shift was represented by a node and it was solved by using a two stage algorithm [8].

Hussain et al.(2011) reported a method to solve the exam timetabling problem based on column generation with graph coloring approach and successfully applied for clustering heuristic to determine the solution quality for exam timetabling problem[9]. In the same time, Razak (2010) has used bipartite graph edge coloring approach to course timetabling. The problem was modeled based on two graphs. The vertices in the first graph represent the lectures who teach a class and the vertices in the second graph are groups of students attending a class and edges represent hard constraint or the meet up between the groups of students attending the class and the lectures who teach the class's respectively [3].

Elghazelet al. (2006) have proposed a new approach of clustering based on a b-coloring of graphs to define a typology of patients. Their contribution in this work was a new clustering algorithm. It was used pair-wise representation, where the objects (hospital stays) are mapped to the nodes of an undirected edge-weighted graph, where the edge weights reflect the dissimilarity between the corresponding pair of nodes. The clustering problem was then formulated as a graph coloring problem [10].

However, Mouna et al. (2011) introduced a new concept for developing the multi-level graph coloring approach and successfully applied it for Bus Driver's timetables. In this context, first objective is to minimize the break time between two travels of one driver. The second objective was working informing between all services. Finally the third objective consists in minimizing the total working hours of all services [11].

\section{PROBLEM DEFINITION}

Huge companies such as telecommunication companies have continuous service all over the day. Comparing with human work capacity, it should divide into three shifts. In such scenario, assign work schedule to each worker and create timetable is challenging task. Most of the current research works has been proposed with either random based optimization algorithms or local optimization algorithms which cannot be used in such difficult scenarios. Especially there are some unpredictable situations such as workers may change their current shifts due to emergencies. In addition uninformed absenteeism, different demand of customers, different religious workers, balancing experience level and skill level of the workers makes the problem more complicate[12][13]. In such kind of scenarios the one who responsible for making timetable has to face difficulties. In our proposed work we can dynamically manage these kinds of especial scenarios.

Our main target is to find high quality unbiased feasible scheduling algorithm and resource assignments under the labor contract rules and satisfying employees as well as employers' requirements and constraints. Here we list down constraints that we were considered.

\section{Constraints}

- $\quad$ Each Customer Officer required working a shift per day.

- Each Customer Officer gets at least one day off per week.

- $\quad$ Each Customer Officer entitled to one day off after a night shift.

- $\quad$ Minimum number of Customer Officers in Peak Time is 8 and Off Peak Time is 5.

- $\quad$ Minimum number of Senior Customer Officers in Peak Time is 3 and Off Peak Time is 2.

- At least one Head Customer Officer in a shift.

- $\quad$ Each Head Customer Officers entitled at least two day off per week.

- Assign skilled Customer Officers to handle Loyalty customers.

- Assign different language Customer Officers per each shift.

- Customer Officers may go on holiday and will not work shift during this time.

- Different religious Customer Officers have different holidays.

- Balancing the work load among Customer Officers.

- Increase maximum number of customer Officers in special holiday days like New Year day, Christmas day etc.

In our proposed work C\#.net as a programming language and SQLServer for handle database management system were used.

\section{METHODOLOGY}

Customer Care roster scheduling represents the important administrative activity in top level telecommunication companies today. Major task is to identify the main areas, main working categories and allocation of recourses in efficient way. Based on requirement analysis, five main categories were identified. They were administrators (employer), head customer officer and other customer officers (employee), customers and other staff members.

\subsection{Client Server Architecture: Roster Analysis Framework}

The new proposed system mainly based on client server architecture. The Employers information stored in database and server is responsible for handles the request of the client to complete some logic task. So, administrator and others uses can directly login to the system through the interface provided and can update and view their records through the system.

As a first step, Administrator in the system is a main user. Administrator is a main handler who controls this system. For reliable handing, administrator appointed sectional heads or Head Customer Officers for each and every department. Sectional heads have a responsibility to maintain their departments' accounts. As a main user, Head Customer Officers creates new accounts for other customer officers in his department. Hence, all the others must register the system 
and need to create their own accounts. Once user gets activated their access, system provides a facility to update their details, apply leaves, apply and confirm OT, view their time tables. So, members must send their leave details through their accounts before creating the roster. In this proposed system, Modified Greedy algorithm is used to create feasible high quality roster.

\subsection{Enhanced Greedy Optimization Algorithm}

In general, a greedy algorithm that follows the heuristic problem solving with locally optimal choice at each stage with the hope of finding a global optimum. Furthermore, it may yield locally optimal solutions that approximate a global optimal solution in a reasonable time. The new Proposed Enhanced Greedy Algorithm has followed five crucial components. They are; Candidate set should be created, selection function should be selects the best candidate to add to the solution, feasibility function should be defined to identify whenever a candidate can be used to contribute to a solution, modified objective function is used to assign a value to local solution, or a partial solution and Solution function helps to identify the complete solution of the intended problem.

The new Proposed Greedy algorithm can be divided into two categories namely; (1) graph coloring approach based ordering for scheduling (2) Clustering [14, 15, 16 and 17].

From a mathematical point of view a graph does not need to be drawn. If we have large number of vertices and edges, it may be make big problem for draw graph. So in real life problems, mathematical methods were used.

One of the definition of graph $\mathrm{G}, \mathrm{G}=(\mathrm{V}(\mathrm{G}), \mathrm{E}(\mathrm{G}))$ consist of two finite sets. V (G), the vertex set of the graph $G$, often denoted by just $\mathrm{V}$, which is a non-empty set of elements called vertices. E (G), the edge set of the graph, often denoted by just $\mathrm{E}$, which a possibly empty set of elements is called edges.

Graphical coloring can be simply explained as coloring the nodes of a graph with the minimum number of colors. In graph theory two adjacent vertices cannot be colored using the same color. The term chromatic number $\chi(G)$ is defined as the minimum number of colors needed for coloring of G. A graph $\mathrm{G}$ is k chromatic, if $\chi(G)=k$ and $G$ is $k$ colorable, if $\chi(G) \leq k$.

Below algorithm used for create adjacency Matrix according to the constraints.

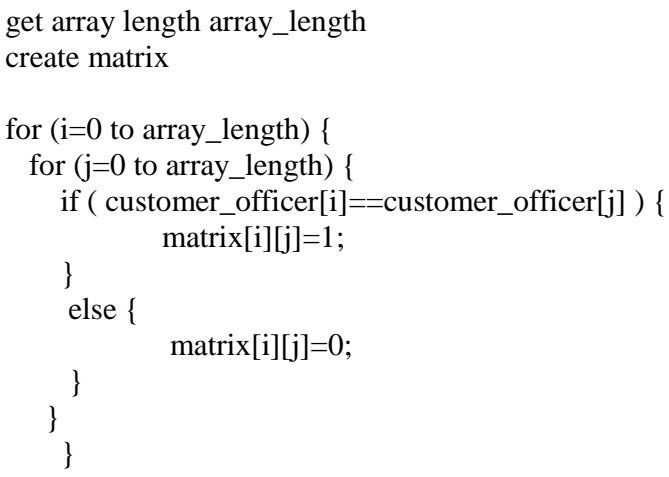

Below algorithm used for create the graph using above adjacency matrix.

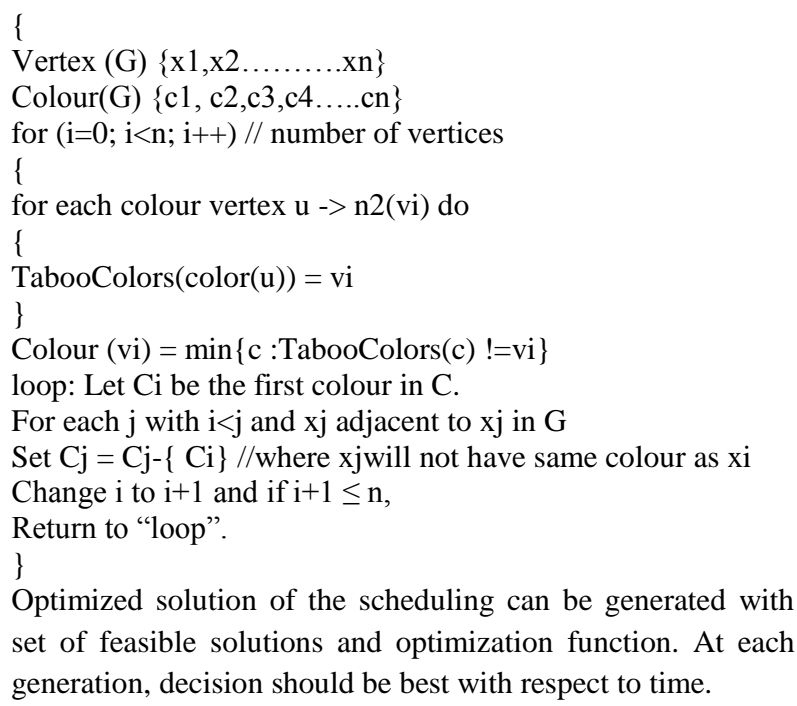
set of feasible solutions and optimization function. At each generation, decision should be best with respect to time.

\section{EXPERIMENTAL RESULTS AND DISCUSSION}

Table 1. Comparison results for selected optimization algorithms

\begin{tabular}{|c|c|c|c|c|}
\hline Days & Count & Algorithm & Solved & $\begin{array}{ll}\text { Avg. } & \text { Time } \\
\text { (Sec.) } & \\
\end{array}$ \\
\hline \multirow[t]{3}{*}{7} & \multirow[t]{3}{*}{1000} & PA & 920 & 1.34 \\
\hline & & SA & 880 & 1.54 \\
\hline & & GA & 800 & 5.00 \\
\hline \multirow[t]{3}{*}{14} & \multirow[t]{3}{*}{1000} & $\mathrm{PA}$ & 940 & 2.68 \\
\hline & & SA & 920 & 5.70 \\
\hline & & GA & 730 & 10.2 \\
\hline \multirow[t]{3}{*}{21} & \multirow[t]{3}{*}{1000} & $\mathrm{PA}$ & 940 & 6.41 \\
\hline & & SA & 910 & 6.96 \\
\hline & & GA & 860 & 15.4 \\
\hline \multirow[t]{3}{*}{30} & \multirow[t]{3}{*}{1000} & $\mathrm{PA}$ & 970 & 21.2 \\
\hline & & SA & 650 & 23.5 \\
\hline & & GA & 640 & 24.1 \\
\hline
\end{tabular}

create array customer_officer 

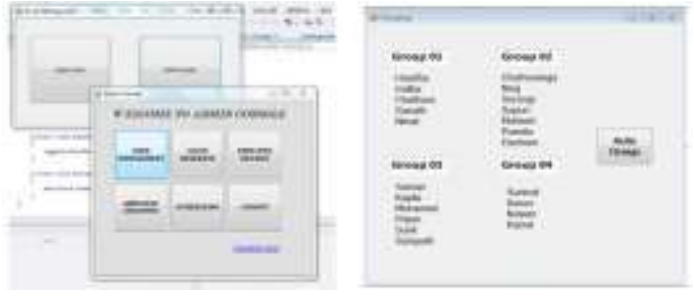

Figure 1. Admin console and grouping interface

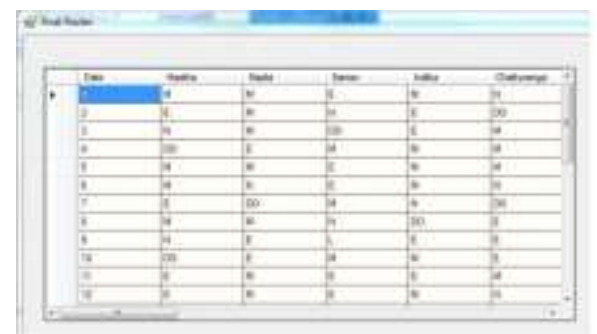

Figure 2. Final roster output

C\#.net programming is used to access information from database. As an initial step, proposed Greedy optimization algorithm is implemented in C\#.net. Detailed information system is created and accessed through SQLServer.

Moreover, optimization with predefined cost function and set of feasible solutions are used to determine the best optimized value. The Problem size is considered as 1000 and compared the proposed greedy solutions with simulated annealing and Genetic algorithm. Optimization is done in weekly in four terms of 7, 14, 21 and 30 days.

Table 1 presents the comparison of proposed results (PA) with simulated annealing (SA) and genetic algorithm (GA) with respect to time in seconds.

As an initial step, average time taken for our proposed greedy is $1.34 \mathrm{sec}$ whereas for $\mathrm{SA}$ it is 1.54 and $\mathrm{GA}$ it is 5.00 seconds. Next, 14 days PA has taken $2.68 \mathrm{sec}$ whereas SA has taken 5.70 and 10.2 for GA respectively. Similarly, for 21 day schedule, 3.21 is for PA, 5.70 for SA and 8.26 for GA. Compared with first two weeks simulation results, third and fourth week simulations result is almost same for PA, SA and GA. According to the results, all the three optimization algorithms holds good to get optimized schedule results in long period simulations. So we can conclude that, it is better to choose greedy or simulated annealing for short period scheduling and can use genetic optimization for long period scheduling's.

\section{CONCLUSION}

Customer hotline roster scheduling is the one of the crucial problem in current telecommunication field. Different kind of special constrains make the problem more and more complicated. Here we proposed new client server roster scheduling framework using graph coloring approach. Proposed frame work is easy to maintain and easy to update for administrator and employee.
In future, enhancements like linking all the databases in different branches for manage all the rosters in every branch in one place can be done to make it easy and high quality.

\section{REFERENCES}

[1] A. Hon, W. Chun, S. Ho, C. Chan, G. Pui, S. Lam, F. Ming, F. Tsang, and J. Wong, Nurse Rostering at the Hospital Authority of Hong Kong, 2000.

[2] S. K. Amponsah, E. Agyeman, and K. G. Okrah, Graph Colouring, an Approach to Nurses Scheduling, Case Study: Ejura District Hospital , Ashanti Region, Ghana, vol. 6, no. 1, pp. 1-5, 2011.

[3] H. A. Razak and Z. Ibrahim, Bipartite Graph Edge Coloring Approach to Course Timetabling, pp. 229-234, 2010.

[4] A. E. P. D, Graph-Coloring for Course Scheduling - A Comparative Analysis based on Course Selection order, pp. 83-88, 2014.

[5] G. L. Prajapati, A. Mittal, R. I. D. Yhuwh, F. Rswlpl, and D. Frpsohwhqhvv, An Efficient Colouring of Graphs Using Less Number of Colours, pp. 666-669, 2012.

[6] A. Elhag and E. Özcan, Expert Systems with Applications A grouping hyper-heuristic framework: Application on graph colouring, Expert Syst. Appl., vol. 42, no. 13, pp. 5491-5507, 2015.

[7] S. Kundu, M. Mahato, B. Mahanty, and S. Acharyya, Comparative Performance of Simulated Annealing and Genetic Algorithm in Solving Nurse Scheduling Problem, vol. I, pp. 19-21, 2008.

[8] J. F. Bard and H. W. Purnomo, Preference scheduling for nurses using column generation q, vol. 164, no. 2, pp. 510-534, 2005.

[9] B. Hussin, A. Samad, and H. Basari, Exam Timetabling Using Graph Colouring Approach, pp. 139-144, 2011.

[10] H. Elghazel, H. Kheddouci, V. Deslandres, A. Dussauchoy, and C. Bernard, A New Graph-Based Clustering Approach : Application to PMSI Data.

[11] M. Mouna, Y. MASMOUDI, and H. C. GIAD, A Multilevel Graph Coloring Approach for the Bus Driver' s Timetables :, pp. 25-31, 2011.

[12] R. M. K. T. Ratnayaka, Z. Wang, S. Anamalamudi, and S. Cheng, Enhanced Greedy Optimization Algorithm with Data Warehousing for Automated Nurse Scheduling System, vol. 2012, no. December, pp. 43-48, 2012.

[13] I. Journal and C. Science, AUTOMATED SYSTEM FOR NURSE SHEDULING USING GRAPH, vol. 2, no. 3, pp. 476-485, 2011.

[14] Rathnayaka, R.M.K.T. ; Wei Jianguo ; Seneviratna, D.M.K.N., Behavior, Economic and Social Computing (BESC), 2014 International Conference on DOI: 10.1109/BESC.2014.7059517

[15] RM Kapila Tharanga Rathnavaka, DMKN Seneviratna, Wei Jianguo, "Grey system based novel approach for stock market forecasting", Grey Systems: Theory and Application, 5(2), 178-193, 2015, Emerald Group Publishing Limited.

[16] Hasitha Indika Arumawadu, RM Kapila Tharanga Rathnayaka, SK Illangarathne, "K-Means Clustering For Segment Web Search Results", International Journal of Engineering Works, 2(8), 79-83, 2015, kwpublisher.com.

[17] Hasitha Indika Arumawadu, RM Kapila Tharanga Rathnayaka, SK Illangarathne, "Mining Profitability of Telecommunication Customers Using K-Means Clustering", Journal of Data Analysis and Information Processing, 3(3), 63, 2015. 